\title{
INFLUENCE OF DISLOCATION INTERACTIONS WITH IMPURITIES ON THEIR TOPOGRAPHICAL IMAGES IN SILICON CRYSTALS
}

\author{
J. Auleytner \\ Institute of Physics, Polish Academy of Sciences \\ Al. Lolników 32/46, 02-668 Warszawa, Poland \\ M.Ya. Skorokinod, L.I. Datsenko and V.I. Khrupa \\ Institute of Semiconductor Plyssics, National Academy of Sciences, Kiev, Ukraine
}

(Received February 5, 1996; revised version June 11, 1996)

Silicon crystals contained copper atoms included by diffusion way during high temperature treatment have been investigated by means of X-ray transmission topography (Lang method). The studies allow us to observe the increase or decrease in the dislocation images widths in dependence on the time of diffusin annealing. In one case, during the more prolonged decoration process a build-up of decorating particles on dislocation occurs with widening of the topographic images of this dislocation. In another case (short time of decorating process) some compensation of defect deformation fields has been noticed (shortening of the mentioned images takes part). The obtained eflects depend not only on the type of intrinsic impurities which take part in forming the Cottrell atmospheres but also on the duration of diffusion annealing. The observed results of interaction of dislocations with impurities have been confirmed by the studies of the integral reflectivity of decorated samples by means of the double-crystal spectrometer.

PACS numbers: 61.10.-i, 61.72.Cc, 61.72.Ff, 81.40.Cd

\section{Introduction}

It is known that point defects interact with deformation fields of dislocations, forming the so-called Cottrell atmospheres around them. This phenomenon alters many strength, electrical, structural and other properties of the crystals. For example, it is known from experiments that diffusion copper into a crystal widens $\mathrm{X}$-ray diffraction images of dislocations [1-3]. The interaction of dislocations with intrinsic point defects as well as with atoms of dopant or with impurities diffusing 
during heat treatments can also influence the integral reflectivity, $R_{\mathrm{i}}$, of the crystals apparently due to changes in values of the coherent and diffuse components of the scattering caused by variations of deformation fields of dislocations. Thus, the measurements of the integral X-ray reflectivity can give useful information about the intensity of interaction between different defects which are important for electronics.

The aim of this paper was to study the effects of interaction of crystal growth dislocations with rapidly diffusing copper atoms by analyzing changes of the X-ray diffraction image widths of dislocations, the integral X-ray reflectivities $R_{\mathrm{i}}$ as well as the values of the static Debye-Waller factor $e^{-L}$.

\section{X-ray topography studies}

For these experiments the $\mathrm{Si}$ crystals obtained by the Czochralski method with a low dislocation density $\left(D_{\mathrm{d}} \approx 5 \times 10^{3} \mathrm{~cm}^{-2}\right)$ were selected. During a growing process they were doped with various types of impurities, e.g. As, P, Ge. The active impurity composition of the real Cottrell atmosphere in the studied crystals is not known unfortunately but we may assume that their formation was determined to a large extent by the impurity whose concentration was the highest. Oxygen can probably be considered as such an impurity because its concentration could reach $1 \times 10^{17} \mathrm{~cm}^{-3}$. Influence of the As and $P$ atoms, whose concentration was about $1 \times 10^{15} \mathrm{~cm}^{-3}$, on formation of the Cottrell atmospheres was less significant. As to Ge atoms $\left(n_{\mathrm{Ge}}=1 \times 10^{18} \mathrm{~cm}^{-3}\right)$ this impurity could prevail in the Cottrell atmospheres.

The samples of all series were decorated at the final stage of thermal treatment at $900^{\circ} \mathrm{C}$ for 1 hour. The topogrammes liave been taken by the Lang method

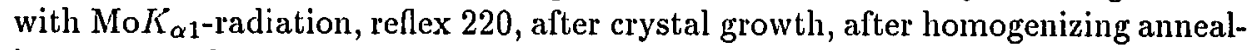
ing at $1250^{\circ} \mathrm{C}$ for 2 hours and after sample decoration with $\mathrm{Cu}$ atoms.

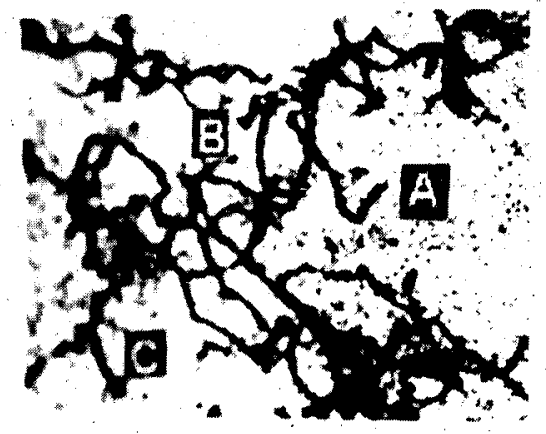

a

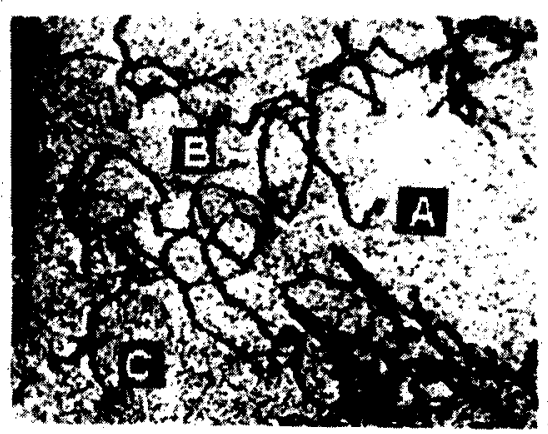

b

Fig. 1. The topogrammes of silicon crystals doped with $P$ impurity after annealing at $1250^{\circ} \mathrm{C}, 2$ hours (a) and after decoration with copper at $900^{\circ} \mathrm{C}, 1$ hour (b). Mo $K_{\alpha 1}$-radiation, the 111 reflex, magnification 26 . The same dislocation image places are noted on both topogrammes by letters $A, B, C$. 
Figure 1a, b shows the topogrammes of samples doped with $\mathrm{P}$ atoms at the initial state (2 hours of annealing at $1250^{\circ} \mathrm{C}$ ) (a) and after decoration of defects (b). One can note the previously unknown effect of narrowing of dislocation image width (marked by letters A, B, C) after a build-up of copper atoms which probably compensate the deformation fields. In order to obtain quantitative confirmation of the results just mentioned an optical comparator was used to compare the widths of dislocation images at fixed points defects structure before and after the decoration process. The average width of images was $29.5 \mu \mathrm{m}$ before decorating, narrowing down to $24.5 \mu \mathrm{m}$ after the copper build-up, i.e. by $17 \%$. Similar results were obtained for crystals doped with arsenic.

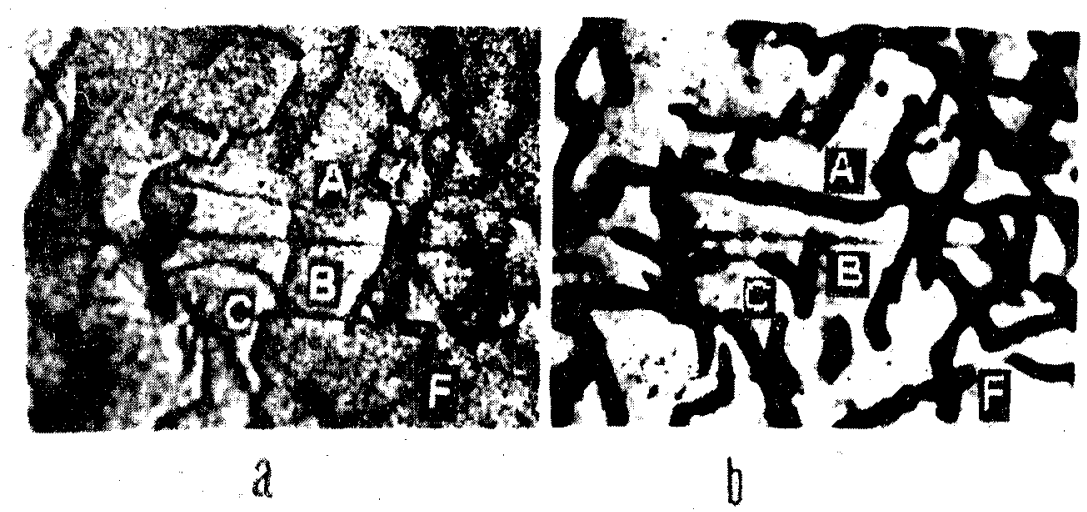

Fig. 2. The topogrammes of silicon crystals annealed with $P$ and Ge impurities (a) and after decoration with $\mathrm{Cu}$ atoms (b). MoKatradiation, the 220 refex, magnification 26. The same dislocation image places are noted on both topogrammes by letters A, B, C, F.

Figure 2a, b shows the results of diffusion annealing on the dislocation crystal structure of the samples doped with $\mathrm{Ge}$ and $\mathrm{P}$. The equivalent details of the dislocation structure images are marked by letters $A, B, C, F$, respectively. It is apparent that the dislocation structure in general did not vary significantly except that new F appeared images (Fig. 2b) which were not visible in the initial state (Fig. 2a) because they did not meet the well-known contrast condition. Unlike the previous experiments now however an opposite tendency is clearly present: the images of dislocations with the edge components of the Burgers vector widen sharply after decoration of dislocation with $\mathrm{Cu}$ atoms. In these crystals an important role in formation of the Cottrell atmospheres is apparently played by the isovalent $\mathrm{Ge}$ atoms which interact with deformation fields of dislocations via elastic forces. The screw dislocations presented in Fig. 2a and 2b (higher up the letter B) and also a part of the bent loop (letter $\mathrm{C}$ ) were not decorated with Cu precipitation. It is known that the screw dislocations do not interact with $\mathrm{Cu}$ atoms in the case of dendritic crystal structure formation [1].

By reviewing the experimental results on Ge-doped crystals (the influence of $P$ and $O$ atoms present in the sample is apparently less significant due to their lower concentration) one can note a substantial increase in the resultant 
structure distortion after $\mathrm{Cu}$ diffusion. This effect has been revealed due to increase in dislocation image widths as well as due to appearance of the dislocation images that were not present in the initial state (Fig. 2a).

The parameters discussed conform that the interaction of rapidly diffusing impurities with the Cottrell atmospheres can result in both extension or significant shrinkage of the eflective deformation fields of dislocations.

\section{Diffractometry studies}

The purpose of these experiments consisted in determination of the quantitative characteristics of structure perfection after $\mathrm{Cu}$ diffusion annealing of various duration. The crystal integral reflectivity $R_{\mathrm{i}}$ in another silicon sample with density of dislocation $D_{\mathrm{d}}=5 \times 10^{4} \mathrm{~cm}^{-2}$ homogeneously distributed within the crystal volume was measured by means of a double-crystal spectrometer $\left(\mathrm{MoK}_{\alpha 1}\right.$-radiation, the 220 and 440 reflections) similar to [4]. Figure 3 shows the dependences of the $R_{\mathrm{i}}$ on the thickness $t$ for a number of crystals. Curve 1 refers to the dislocation - free crystal (for comparison purposes); the curves $2-4$ are for the samples

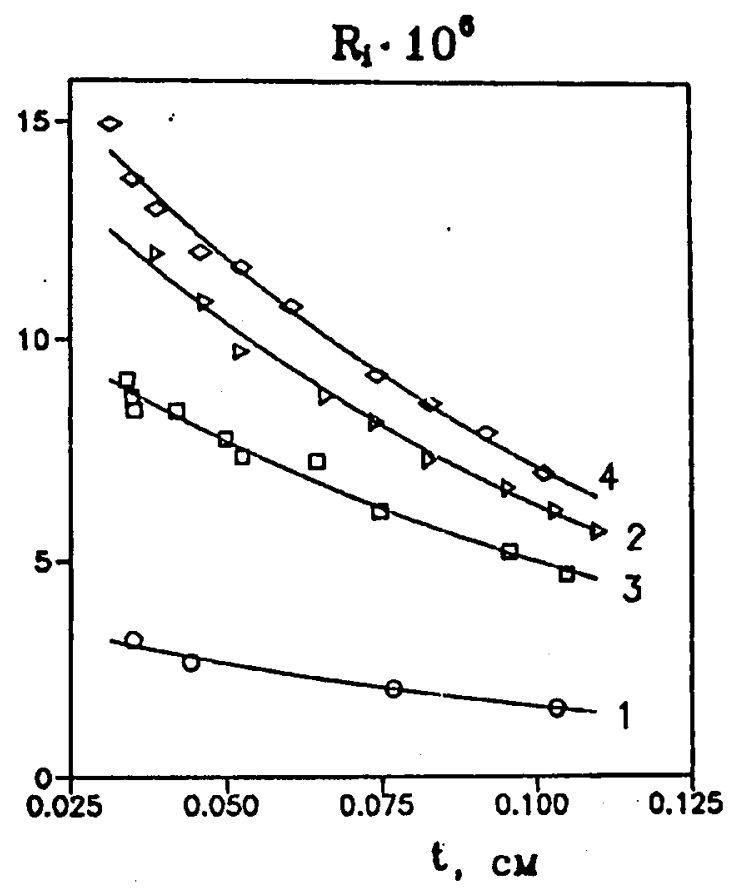

Fig. 3. The dependence of the integral crystal reflectivity $R_{\mathrm{i}}$ on the sample (series 2) thickness with fixed dislocation density (samples 2-4). 1 - the perfect crystal for comparison (calculated and measured). 2 - the initial stage of studied crystal, 3 - crystal decorated with $\mathrm{Cu}$ for 3 hours at $900^{\circ} \mathrm{C}(2,3,4$ measured dots only). 
with the dislocation density indicated. It is possible to note that all three samples (2 - initial state; 3,4 - decorated at $900^{\circ} \mathrm{C}$ for 1 and 3 hours, respectively) are characterized by the $R_{\mathrm{i}}$ values much higher than the ones of the reference crystal 1 .

The total crystal reflectivity for the crystals with defects, as it is known, consists of the diffuse $R_{\mathrm{D}}$ and the coherent $R_{\mathrm{B}}$ terms: $R_{\mathrm{i}}=R_{\mathrm{D}}+R_{\mathrm{B}}$. One should notice that the character of the integral reflectivity increments $\Delta R_{\mathrm{i}}$ is different in samples 3 and 4 in relation to sample 1 . It is 1.3 times lower after diffusion annealing for 1 hour than in the undecorated sample 2, while after longer annealing for 3 hours it is 1.2 times higher than that in the sample 2 . With reference to results of topographic study mentioned earlier such phenomena in scattering of X-rays can be explained, on the one hand, by certain compensation of deformation fields of dislocation by copper solution in silicon in the first case (1 hour), and, on the other hand, by increase of the resultant lattice distortion during the built-up particles of the $\beta$-CuSi at defects [3] after 3 hour annealing when the total quantity of copper injected into the sample 4 is larger than that in the sample 3.

The following quantitative interpretation can be given to the observed diffraction phenomena. As it was shown earlier $[4,5]$ the value of increment $\Delta R_{\mathrm{i}}$ due to diffuse scattering on dislocations can be described by the following expression:

$$
\Delta R_{\mathrm{i}}=R_{\mathrm{i}}-R_{\mathrm{ip}}=Q \ell \gamma^{-1} \exp (-\mu l)[1-\exp (-2 L)],
$$

where $R_{\mathrm{i}}$ - the experimental value of integral reflectivity for a real crystal, while $R_{\text {ip }}$ is the same parameter for a crystal with a perfect structure (Fig. 3, curve 1); $Q$ is a parameter characterizing the kinematical scattering ability of unite volume for the given reflection. $\gamma, t$ and $\mu$ stand for cosine of the Bragg angle, thickness of a sample and linear coefficient of photoelectric absorption, respectively.

The factor $L$ for crystals with uniform distribution of dislocations is proportional to the dislocation density $[4,5], L \approx r^{2} D_{\mathrm{d}}$, where $r$ is a radius of the region of strong distortions around dislocations, $r=(H b) \Lambda^{2} D_{\mathrm{d}} / 4 \pi$ and $H, b, \Lambda$ are respectively a diffraction vector, the Burgers vector and an extinction length. By measuring the value of $\Delta R_{\mathrm{i}}$ arising due to diffuse scattering on dislocations it is possible to determine the $L$ magnitude and accordingly estimate the value of $D_{\mathrm{d}}$. Considering the demonstrated influence of interaction of impurity atoms with deformation fields of dislocations on X-ray scattering we should refer its values of the $L$ and $D_{\mathrm{d}}$. The calculations of these parameters based on the $\Delta R_{\mathrm{i}}$ for the 400 reflection (MoK $K_{\alpha}$-radiation) in the samples 2,3 and 4 provided the following results: $L_{2}=0.043, L_{3}=0.035, L_{4}=0.058$ and for the $D_{\mathrm{d}}: D_{\mathrm{d} 2}=2 \times 10^{5} \mathrm{~cm}^{-2}$, $D_{\mathrm{d} 3}=1.6 \times 10^{5} \mathrm{~cm}^{-2}, D_{\mathrm{d} 4}=2.7 \times 10^{5} \mathrm{~cm}^{-2}$. The obtained $D_{\mathrm{d}}$ values are close to $5 \times 10^{5} \mathrm{~cm}^{-2}$ derived from the density of etch pits and reflect the result of various interactions between dislocations and impurities.

Thus, the X-ray diffraction studies of the reflectivity variation and the widths of topographical images of dislocations in crystals when the $D_{\mathrm{d}}$ value is constant enable us to perform a quantitative estimation of the degree of interaction of impurities with growth dislocations. The compensating action of rapidly diffusing impurities on the effective size of the Cottrell atmosphere was found to be similar for such electrically active impurities as As and $\mathrm{P}$ despite a significant difference in the size of their atoms. Upon diffusion of copper into a sample doped with 
isovalent germanium a continuous series of solid solutions with silicon can form. In consequence the favourable conditions for separation of decorating particles are formed. These particles enlarge the volume of distorted lattice. In samples taken from the same ingot the compensation effect is observed after the short (1 hour) diffusion annealing, when the integral reflectivity and the magnitude of the static Debye-Waller factor is significantly reduced in comparison with a non-decorated crystal. We believe that the methodical approach suggested can be a useful supplement to the method which evaluates the gettering efficiency of impurities by the analysis of the ratio of intensities of the soft and hard components of $\gamma$-radiation of the radioactive $\mathrm{Co}^{57}$ impurity [6].

\section{Conclusions}

The non-destructive methods applied to the studies, presented in this paper, allow us to investigate the dislocation structure changes by the $\mathrm{Cu}$ diffusion into silicon crystals during high temperature annealing. Influence of the different types of impurities doped into a crystal lattice during a growing process on the dislocation was shown. This influence was different in the case of isovalent Ge and in As and $P$ electrical active impurities.

\section{References}

[1] H. Tomitsu, J. Phys. Soc. Jpn. 40, 505 (1976).

[2] J. Auleytner, J. Morawiec, V. Khrupa, L. Datsenko, M. Skorokhod, Materiaty 3 Krrajowego Sympozjum Użylkowników Promieniowania Synchrotronowego, Ed. E. Sobczak, Fundacja im. Wojciecha Świętosławskiego na Rzecz Wspierania Nauki i Rozwoju Potencjału Naukowego w Polsce, Warszawa 1995, p. 34 (in Polish).

[3] E. Nes, J. Washburn, J. Appl. Phys. 43, 2005 (1972).

[4] L.I. Datsenko, V.B. Molodkin, M.E. Osinovskii, Dynamical X-ray Scaltering of Deformed Crystals, Naukova Dumka, Kiev 1995 (in Russian).

[5] V.F. Machulin, V.I. KLhrupa, X-ray Diagnostics of Structural Perfection of Weak Deformed Cristal, Naukova Dumka, Kiev 1995 (in Russian).

[6] D. Gilles, Solid State Phenomena 32-33, 57 (1993). 